\title{
VEGF and TNF- $\alpha$ Responses to Acute and Chronic Aerobic Exercise in the Patients with Multiple Sclerosis
}

\author{
Shoeib Rezaee ${ }^{1}$, Sedighe Kahrizi ${ }^{1,{ }^{*}}$, Seyed Massood Nabavi ${ }^{2}$ and Mehdi Hedayati ${ }^{3}$ \\ ${ }^{1}$ Physical Therapy Department, Faculty of Medical Sciences, Tarbiat Modares University, Tehran, Iran \\ ${ }^{2}$ Regenerative Biomedicine Department, Cell Science Center, Royan Institute for Stem Cell Biology and Technology, Tehran, Iran \\ ${ }^{3}$ Cellular and Molecular Endocrine Research Center, Research Institute for Endocrine Sciences, Shahid Beheshti University of Medical Sciences, Tehran, Iran \\ "Corresponding author: Physical Therapy Department, Faculty of Medical Sciences, Tarbiat Modares University, Tehran, Iran. Email: kahrizis@modares.ac.ir \\ Received 2019 November 30; Revised 2020 April 25; Accepted 2020 April 26
}

\begin{abstract}
Background: Aerobic exercise has been shown to decrease the functional disorders related to multiple sclerosis (MS). Nevertheless, the effect of aerobic exercise training on the cytokines and neuroprotective mediators associated with the disease activity has remained relatively unknown.

Objectives: The present research was conducted to evaluate the responses of the vascular endothelial growth factor (VEGF) and tumor necrosis factor-alpha (TNF- $\alpha$ ) to single-bout and six-week aerobic exercise training in the patients with MS.

Methods: Totally, 20 subjects ( $(n=10)$ patients with MS and $(n=10)$ controls) who were matched in terms of gender, weight, body mass, and age were included in the study. Participants received aerobic exercise training at approximately $60 \%$ of $\mathrm{VO}_{2 \max }$ in 30 -min sessions 3 times a week for six weeks. Serum concentrations of the VEGF and TNF- $\alpha$ were analyzed pre-and post-exercise training (in the first session and after 18 sessions of the study).

Results: Serum concentration of the TNF- $\alpha$ decreased significantly after a single bout and 6-week aerobic exercise training $(\mathrm{P}=$ 0.002 and $\mathrm{P}=0.005)$ in the intervention group. Serum concentration of the VEGF increased significantly only after exercise in the first and 18th sessions in the intervention group $(\mathrm{P}=0.017$ and $\mathrm{P}=0.022)$.

Conclusions: Our results revealed that exercise may influence the VEGF regulation in patients with MS. Although, further research is needed to determine the effect of exercise on the immune variables in patients with MS.
\end{abstract}

Keywords: Multiple Sclerosis, VEGF, Aerobic Exercise, TNF- $\alpha$

\section{Background}

Multiple sclerosis (MS) is a chronic inflammatory demyelinating disease of the brain and spinal cord characterized by various forms of inflammation, axonal, and demyelination damage. Cytokines have a critical role in the development of MS and are considered an important factor in the treatment interventions. Tumor necrosis factoralpha (TNF- $\alpha$ ) plays an outstanding role in the progression of demyelination and axonal loss endured by the patients with MS (1). In addition, TNF- $\alpha$ is made by various cells, containing immune cells and neurons. There is a great body of evidence representing the role of TNF- $\alpha$ in MS. For instance, augmented amounts of TNF- $\alpha$ have been found in the lesions present in the central nervous system (CNS) as well as in the serum of patients with MS. Augmented concentrations of TNF- $\alpha$ are also associated with the intensity and development of the disease. These robust findings have been reinforced by the effective treatment plan of blocking TNF- $\alpha$ in mouse models of MS (2). Vascular en- dothelial growth factor(VEGF) is involved in the migration, proliferation and matrix degradation, creation of vascular network as well as formation and release of nitric oxide (NO) in the endothelial cells. Angiogenesis is mediated by a number of molecules that can be considered in emerging treatments for the patients with MS. Apparently, angiogenesis is needed in response to an augmented demand for the nutrients and oxygen for impaired neural cells and renovation of the cells in the plaques. Thus, angiogenesis is useless during chronic MS because of axonal activity, repair of cell disparity, and cellular irregularities resulting in the vasoconstriction of newly formed blood vessels and hindering the blood flow. Therefore, studies on the trophic factors that stimulate angiogenesis in the brain can be valuable (3). Another prospective candidate suggested to mediate the effects of exercise on the brain is VEGF (4). Additionally, VEGF contributes to initiating the hippocampal neurogenesis and angiogenesis; once peripheral VEGF is hindered, neurogenesis is also inhibited unexpectedly post-

Copyright (c) 2020, Asian Journal of Sports Medicine. This is an open-access article distributed under the terms of the Creative Commons Attribution-NonCommercial 4.0 International License (http://creativecommons.org/licenses/by-nc/4.0/) which permits copy and redistribute the material just in noncommercial usages, provided the original work is properly cited. 
exercise (5). To the best of our knowledge, VEGF response to exercise has not been investigated in patients with MS so far. Therefore, further study is needed in this regard to clarify that whether exercise can improve the neurogenesis factors in patients with MS.

Aerobic exercise has been revealed to adjust the immunological responses during the production of the cytokines in short-term sessions of exercise in healthy people (6). Given the health benefits related to consistent physical activity, additional research is needed to interpret the effect of exercise on immune responses in MS. It has been suggested that the immediate discharge of cytokines through short-term exercise can lead to the preservation of a homeostatic immune setting (7). Moreover, numerous proteins secreted in response to cytokine changes are inhibitors or free-radical hunters that decrease body lesions by stimulated neutrophils (7). Accordingly, a distinct session of exercise is a moderate corporeal stimulator that applies a pattern of special effects on the immune frameworks $(8,9)$. The effect of physical activity on inflammatory conditions in the patients with MS stays unresolved with various consequences. Particularly, previous studies have shown that after participation in physical activity training program, serum pro-inflammatory cytokine levels remain unchanged $(9,10)$ are reduce $(11)$, and increase (12) in patients with MS. Previous documents have proposed that exercise pressure and training grade could have immunomodulatory effects; however, these results need to be verified. Further study is required to entirely determine the effect of exercise training on immune condition in patients with MS.

\section{Objectives}

Consequently, the primary objective of this research is to investigate the VEGF and TNF- $\alpha$ responses to acute and chronic exercise in the patients with MS due to the important role of TNF- $\alpha$ and VEGF in the pathogenesis of MS. So, the following hypothesis was put forth: Aerobic exercise reduces the serum concentration of TNF- $\alpha$ and increases the serum concentration of VEGF in the patients with MS.

\section{Methods}

\subsection{Participants}

Twenty subjects with clinically diagnosed relapsingremitting MS (RRMS) in accordance with the 2010 McDonald's criteria (13), who were safe and had slight to mild defect [Expanded Disability Status scale (EDSS) score of 0 - 4] were recruited in this study. Participants diagnosed with cardiovascular diseases, diabetes, a history of alcohol abuse, using steroid treatment in the last 2 months, and musculoskeletal problems were excluded from the present study (14). Furthermore, participants who had experienced a relapse or were unable to complete the whole 30-min cycle ergometer test were also excluded.

This single-blinded randomized controlled trial study was approved by the local Ethics Committee of Tarbiat Modares University and was registered in the Iranian Registry of Clinical Trials (IRCT) with certification number of IRCT20090203001637N5. A detailed explanation about the procedures of the study was given to all the subjects, and informed written consent was obtained from all of them prior to participating in the study.

\subsection{Experimental Design}

Subjects were randomly divided into two groups: control or non-exercise group $(\mathrm{n}=10)$ and aerobic training treatment group $(n=10)$. Subjects were also matched in terms of gender, body mass index (BMI), and age in both control and treatment groups. The treatment included a six-week aerobic training plan in which the participants practiced on a bicycle three times a week for 30 minutes at $60 \%\left(\mathrm{VO}_{2 \max }\right)$. Every exercise session involved a 5-min warm-up at a self- determined convenient workload pursued by 30 minutes of cycling at $60 \%$ of $\mathrm{VO}_{2 \max }$ (three days/week). A six-week training plan was designated to obtain an appropriate provocation to change the cardiovascular capability, neuroprotection, and immunomodulation in patients with MS (15).

\subsection{Graded Exercise Testing}

Subjects referred to the test center for assessment at an identical hour of the day (8 - 10 AM) and subsequently avoided performing exercise, and consuming foods or alcohol for the past 12 hours. Participants were acquainted with the testing apparatus and simultaneously completed a Graded Exercise testing (GXT). Then, the GXT was started with 5 min warmup (desired RPM and workload), pursued by a 5 - 20 Watt raise in the intensity each lasting for two minutes as far as the subject attained a restricted maximum sign or $85 \%$ of predictable maximal heart rate terminus. Blood pressure and heart rate were measured throughout the assessment. Results obtained from the GXT were considered to manage the personal training workloads during the study (16). In each training session, the level of the resistance cycle ergometer was increased to reach the target intensity in order to achieve the progressive exercise protocol.

\subsection{Outcome Measures}

\subsubsection{Blood Sampling and Assessment}

Blood samples ( $6 \mathrm{~mL}$ ) were collected from the subjects before and immediately after the first and 18th sessions of 
training for the treatment and control groups. These sessions were arranged at the same time for all the participants (8:30 to 9:30 AM) to handle the circadian rhythm of the assessed biological factors. Plasma samples were instantly centrifuged at $3000 \mathrm{~g}$ for $15 \mathrm{~min}$ at $4^{\circ} \mathrm{C}$ and then were stored at $-80^{\circ} \mathrm{C}$ for subsequent analyses. Samples were instantly centrifuged at $3000 \mathrm{~g}$ for $15 \mathrm{~min}$ at $4^{\circ} \mathrm{C}$ and later, were kept at $-80^{\circ} \mathrm{C}$ for further analyses. Serum concentrations of the VEGF and TNF - $\alpha$ were determined by the enzyme-linked immunosorbent assay (ELISA) kits according to the manufacturer's instructions. The VEGF level was measured by the ZellBio GmbH kit (Ulm, Germany). Also, the TNF- $\alpha$ level was determined by the Diaclone kit (Besancon, France). The intra-assay coefficient of variation for the VEGF and TNF- $\alpha$ was equal to 6.9 and $5.8 \%$, correspondingly, arranged by the manufacturer. The special sensitivities of VEGF and TNF- $\alpha$ were equal to $10 \mathrm{ng} / \mathrm{mL}$ and $8 \mathrm{pg} / \mathrm{mL}$, respectively, as provided by the manufacturer.

\subsection{Statistical Analysis}

The Shapiro-Wilk test showed that the data were normally distributed in both groups $(P=0.7)$. Significance level was accepted at $\mathrm{P}<0.05$ and the data were analyzed using the independent $t$-test followed by the post hoc Bonferroni correction for the baseline group differences in the demographic data. Repeated measures Analysis of Variance (ANOVA) was used in each group to measure VEGF and TNF- $\alpha$ before and immediately after the first and 18th sessions in both groups (control vs. aerobic training group). Mixed ANOVA and post hoc tests were used to assess the interaction effects of time (before and immediately after the first and 18th sessions) as within-subject factors and groups (control vs. aerobic training group) as the betweensubject factor on TNF- $\alpha$ and VEGF. All the statistical analyses were performed using the SPSS 24 software. All the data were presented as means $\pm \mathrm{SD}$.

\section{Results}

The baseline demographic data (age, BMI, gender, EDSS score, and baseline blood factor) were similar in the two study groups and no significant differences were found between the groups $(\mathrm{P}>0.05)$ (Table 1$)$.

\subsection{VEGF Concentration in the Serum}

The results showed that the effect of time $(F=14.77$ and $\mathrm{P}=0.001)$ was significant, but the effect of group factor was not significant $(\mathrm{F}=2.38$ and $\mathrm{P}=0.14)$. Also, the interaction effect between time and group showed significant changes $(\mathrm{F}=17.33$ and $\mathrm{P}=0.001)$. Independent $t$-test showed that the mean serum concentration of VEGF was not significantly different between the two groups before the intervention at the first session $(\mathrm{t}(18)=0.33$ and $\mathrm{P}=0.7)$. No significant difference was found in the serum concentration of VEGF in the control group at different times $(\mathrm{t}(18)=0.34$ and $\mathrm{P}=$ 0.7). The amount of VEGF increased significantly after exercise in the first and 18th sessions in the exercise group in comparison with the control group $\mathrm{t}(18)=2.61$, $(\mathrm{P}=0.017)$, $t(18)=2.51,(P=0.022)$. However, there was no significant change in the serum concentration of VEGF before the exercise in the first and 18 th sessions $(\mathrm{t}(18)=0.41$ and $\mathrm{P}=0.6)$, (Figure 1).

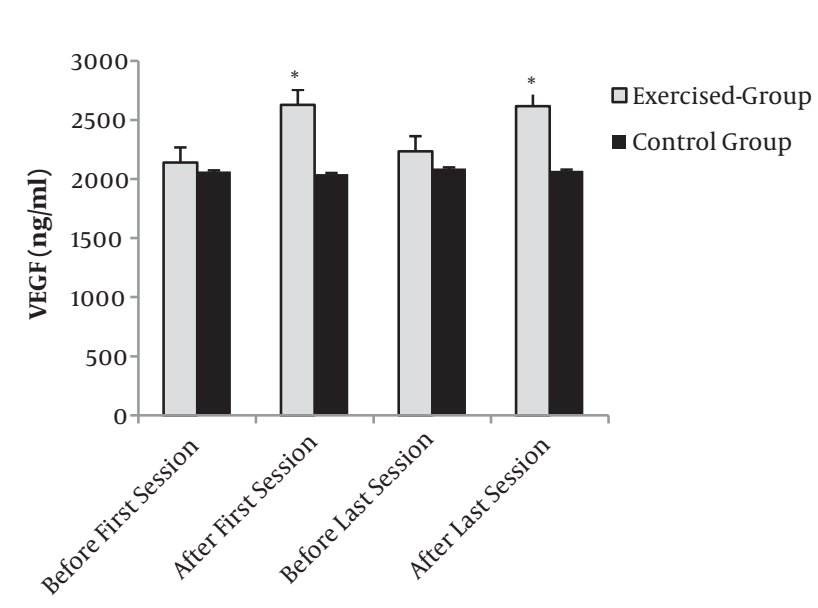

Figure 1. Evaluation of mean VEGF between two groups of exercise and control. *, indicates significant differences $(\mathrm{P}<0.05)$ between the exercised and control group. Data are expressed as mean \pm standard deviation.

\subsection{TNF- $\alpha$ Concentration in the Serum}

The results showed that the effect of time $(\mathrm{F}=21.99$ and $\mathrm{P}=0.0001)$, group $(\mathrm{F}=19.07$ and $\mathrm{P}=0.0001)$, and the interaction effect between time and group were significant $(\mathrm{F}=$ 21.70 and $P=0.0001)$. Independent t-test showed that the mean serum concentration of TNF- $\alpha$ was not significantly different between the two groups before the intervention in the first session $(\mathrm{t}(18)=0.07$ and $\mathrm{P}=0.9)$. No significant difference was found in the serum concentration of TNF$\alpha$ in the control group at different times $(t(18)=0.06$ and $\mathrm{P}=0.8$ ). Pairwise comparison of mean serum concentration of TNF- $\alpha$ between two groups in the first session after the exercise showed that the amount of TNF- $\alpha$ in the exercise group was considerably lower than that of the control group $(t(18)=3.6$ and $P=0.002)$. In the 18th session, the amount of TNF- $\alpha$ decreased significantly before and after the intervention in the treatment group compared to the control group $(\mathrm{P}=0.004$ and $\mathrm{P}=0.003)$. Also, a significant decrease was found in the TNF- $\alpha$ level before the exercise in the first and 18th sessions $(\mathrm{t}(18)=3.5$ and $\mathrm{P}=0.004$ ), (Figure 2). 


\begin{tabular}{|c|c|c|c|c|}
\hline & $\operatorname{Exercised}(\mathrm{N}=10)$ & Control $(N=10)$ & PValue & $t(18)$ \\
\hline Age & $28.9 \pm 3.3$ & $28.5 \pm 2.9$ & 0.7 & 0.28 \\
\hline BMI & $24.4 \pm 1.4$ & $24.3 \pm 1.2$ & 0.9 & 0.09 \\
\hline EDSS & $2.2 \pm 0.4$ & $2.6 \pm 0.5$ & 0.7 & 0.18 \\
\hline VEGF, ng/mL & $2140 \pm 645$ & $2063 \pm 339$ & 0.7 & 0.33 \\
\hline TNF- $\alpha, \mathbf{p g} / \mathbf{m L}$ & $19.06 \pm 4.3$ & $19.07 \pm 1.8$ & 0.9 & 0.07 \\
\hline Gender (M/F), n & $4 / 6$ & $4 / 6$ & 1 & 0.00 \\
\hline
\end{tabular}

${ }^{\mathrm{a}}$ Values are expressed as mean $\pm \mathrm{SD}$.

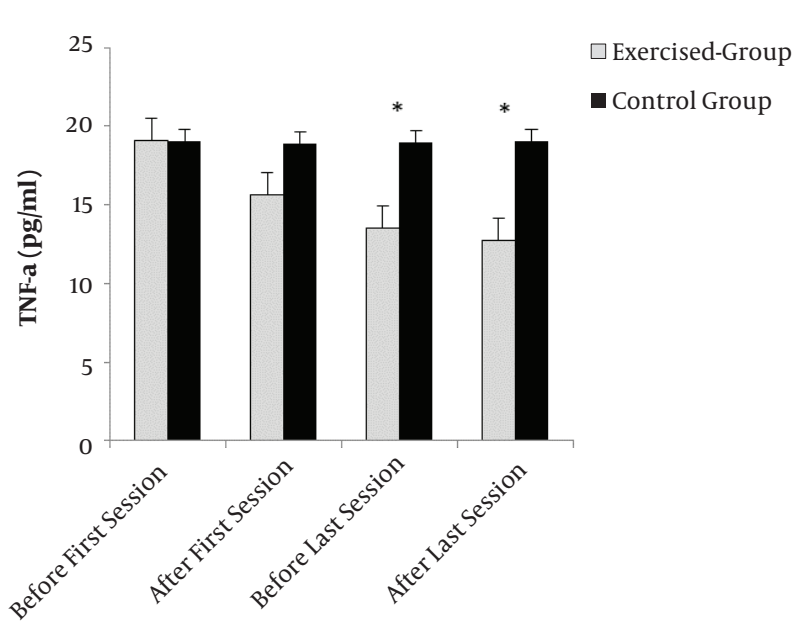

Figure 2. Comparison of mean TNF- $\alpha$ between two groups of exercise and control. *, indicates significant differences $(\mathrm{P}<0.05)$ between exercised and control groups. Data are expressed as mean \pm standard deviation

\section{Discussion}

There are limited clinical researches regarding the effect of exercise training on the production of cytokines in the patients with MS. Exercise has been introduced as a factor that raises the circulation of immune cells, causing physiological signaling and participation in the neuroprotection and plasticity of the nervous system (9). Our results showed that exercise training can influence the TNF- $\alpha$ and VEGF concentrations. This study is the first study investigating the VEGF responses to exercise in patients with MS. Aerobic exercises can influence the angiogenesis process by increasing the expression of angiogenic factors such as VEGF as well as decreasing the expression of angiostatic factors like endostatin $(17,18)$. The results showed that the VEGF level increased after a single bout of exercise but remained unchanged after 6 weeks. There are controversial findings regarding the effect of exercise on serum concentration of VEGF. Van Craenenbroeck et al. (19), have shown that serum concentration of VEGF was increased follow- ing intensive exercise by cycle ergometer in healthy subjects. However, Suhr et al. (20), have reported no change in the serum concentration of VEGF after short-term highintensity cycling exercise in healthy subjects. The difference in the results probably can be ascribed to training variables (intensity, duration, type of physical exercise) and subject's status (age, fitness, and body composition) $(17,20,21)$. The acute increase in the serum concentration of VEGF in our study may be attributed to hypoxia, shear stress, muscle contraction, and tension (22), while the unchanged level of VEGF following exercise training may be due to VEGF binding to the receptors on endothelial cells stimulating the angiogenesis in the muscles (19). It has been reported that VEGF transcription level in skeletal muscle is the most essential factor in the regulation of serum concentration of VEGF (20). As a molecule that generally promotes proliferation, migration, and tube formation of the endothelial cells, VEGF is the main stimulus of angiogenesis. In the chronic stages of MS, VEGF has a neuroprotective role and promotes the proliferation and survival of neural cells. The VEGF level decreases in the patients with MS and animals with Experimental Autoimmune Encephalomyelitis (EAE) (21).

Results of the present study showed that 6-week exercise training caused a significant reduction in the TNF- $\alpha$ level. Previous researches have demonstrated unreliable outcomes regarding the effect of training on TNF- $\alpha$ level in patients with MS. For example, Castellano et al., (12) and Deckx et al. (23), have reported a rise and a reduction in the TNF- $\alpha$ level, correspondingly. On the contrary, AlvarengaFilho et al. (24), and Kjolhede et al. (25), have indicated no variation after the training program. These differences in the results might imply the pleiotropic role of TNF- $\alpha$ (12, 25). Herein, the TNF-a response was measured after the single session of exercise. Our results showed that TNF- $\alpha$ level decreased significantly post-exercise, which is consistent with previous findings $(12,25)$. Our results are not consistent with the study by Heesen et al. (15), who stated augmented TNF- $\alpha$ level immediately after the exercise (30 min of cycle ergometer at $\left.60 \% \mathrm{VO}_{2 \text { peak }}\right)$. However, previous re- 
ports have shown the detrimental effect of TNF- $\alpha$ on the myelin sheath and blood-brain barrier, and (26)TNF- $\alpha$ level is associated with the remission of the disease and stimulates the remyelination (27). This effect likely results from the conflicting properties of TNF-receptors: p75 with neuroprotection part or p55 with apoptotic part (28). TNF- $\alpha$ involves an extensive range of biological purposes and is the main factor influencing the pathogenesis of chronic inflammation and autoimmune syndromes. Therefore, antiTNF therapies have been developed for the treatment of autoimmune and inflammatory diseases (29). Numerous clinical researches have suggested the significant role of TNF in MS. Patients with chronic progressive MS have been shown to have elevated TNF levels in the cerebrospinal fluid (CSF) and active lesions. The TNF level is associated with the presence and grade of disability in patients with MS (30).

Previous studies have shown that these methods of aerobic exercise cause an appropriate provocation to change cardiovascular capability and immunomodulation in patients with MS (15). A cycling trial is a low-impact exercise that uses smooth activities to reinforce the joints and muscles without putting great force on them. Furthermore, it is safer than outdoor cycling. Outdoor cycling would be a good approach to exercise, but it is accompanied by definite dangers, such as careless drivers, bumpy or slippery street surfaces, and hot weather (31). Designing this exercise is easy for the patients as well as therapists. Individuals can easily simulate such a practice at home by learning to estimate the maximum heart rate according to their age.

\subsection{Study Limitations}

There were some limitations to this study. Firstly, the concentrations of VEGF and TNF- $\alpha$ were not determined in the CSF concurrently pre- and post-exercise, and it would be worthy to compare the concentrations of VEGF and TNF$\alpha$ in both CSF and serum simultaneously in the future research. Secondly, to the best of our knowledge, this study is the first exercise intervention study on MS that investigated the VEGF level and our results highlight the need for further studies on this factor with larger sample sizes.

\subsection{Conclusions}

There is incomplete evidence on the effect of training on the immune responses that has been reported to influence the disease condition in the RRMS. Our results suggested that a single bout of exercise reduces the amount of TNF- $\alpha$ and increases the amount of VEGF in the patients with MS, a decrease was also observed in the baseline TNF$\alpha$ level after six weeks of training in the treatment group. Accordingly, further research is required to obtain perfect and inclusive results regarding the VEGF and TNF- $\alpha$ responses to aerobic exercise in MS and its associations on disease condition.

\section{Acknowledgments}

This study was supported by Medical Sciences of Tarbiat Modares University, Tehran, Iran, and Royan Institute for Stem Cell Biology and Technology, Tehran, Iran. The authors would like to thank persons who participated in the study.

\section{Footnotes}

Authors' Contribution: SR developed the original idea and the protocol, abstracted and analyzed data, and wrote the manuscript. SK developed the original idea and material, study supervision and critical revision of the manuscript for important intellectual content. SN performed study supervision, administrative, technical, and material support. MH analyzed blood samples.

Clinical Trial Registration Code: The clinical trial registration code was IRCT20090203001637N5.

Conflict of Interests: There is no conflict of interest.

Ethical Approval: This single-blinded randomized controlled trial study was approved by the local Ethics Committee of Tarbiat Modares University (https://en.irct.ir/user/trial/1100/view).

Funding/Support: This study was supported by Medical Sciences of Tarbiat Modares University, Tehran, Iran and Royan Institute for Stem Cell Biology and Technology.

Informed Consent: A detailed explanation about the procedures of the study was given to all the subjects and informed written consent was obtained from all of them prior to participating in the study.

\section{References}

1. Coles AJ, Cox A, Le Page E, Jones J, Trip SA, Deans J, et al. The window of therapeutic opportunity in multiple sclerosis: Evidence from monoclonal antibody therapy. J Neurol. 2006;253(1):98-108. doi: 10.1007/s00415-005-0934-5. [PubMed: 16044212].

2. Sharief MK, Hentges R. Association between tumor necrosis factoralpha and disease progression in patients with multiple sclerosis. N Engl J Med. 1991;325(7):467-72. doi: 10.1056/NEJM199108153250704. [PubMed: 1852181].

3. Iacobaeus E, Amoudruz P, Strom M, Khademi M, Brundin L, Hillert J, et al. The expression of VEGF-A is down regulated in peripheral blood mononuclear cells of patients with secondary progressive multiple sclerosis. PLoS One. 2011;6(5). e19138. doi: 10.1371/journal.pone.0019138. [PubMed: 21573104]. [PubMed Central: PMC3089609].

4. Yau SY, Lau BW, So KF. Adult hippocampal neurogenesis: A possible way how physical exercise counteracts stress. Cell Transplant. 2011;20(1):99-111. doi: 10.3727/096368910X532846. [PubMed: 20887683]. 
5. Fabel K, Fabel K, Tam B, Kaufer D, Baiker A, Simmons N, et al. VEGF is necessary for exercise-induced adult hippocampal neurogenesis. Eur J Neurosci. 2003;18(10):2803-12. doi: 10.1111/j.1460-9568.2003.03041.x. [PubMed: 14656329].

6. Mackinnon LT. Advances in exercise immunology. United Kingdom: Human Kinetics; 1999. doi: 10.1201/9781420041798.ch1.

7. Moldawer LL. The beneficial role ot cytokines, particularly interleukin-1, in the host responce to injury, infection, and inflammation. Cytokines Health Di. 1992:217-34.

8. Sprenger H, Jacobs C, Nain M, Gressner AM, Prinz H, Wesemann W, et al. Enhanced release of cytokines, interleukin-2 receptors, and neopterin after long-distance running. Clin Immunol Immunopathol. 1992;63(2):188-95. doi: 10.1016/0090-1229(92)90012-d. [PubMed: 1611721].

9. Schulz KH, Gold SM, Witte J, Bartsch K, Lang UE, Hellweg R, et al. Impact of aerobic training on immune-endocrine parameters, neurotrophic factors, quality of life and coordinative function in multiple sclerosis. J Neurol Sci. 2004;225(1-2):11-8. doi: 10.1016/j.jns.2004.06.009. [PubMed: 15465080].

10. Bansi J, Bloch W, Gamper U, Kesselring J. Training in MS: Influence of two different endurance training protocols (aquatic versus overland) on cytokine and neurotrophin concentrations during three week randomized controlled trial. Mult Scler. 2013;19(5):613-21. doi: 10.1177/1352458512458605. [PubMed: 22936334].

11. Golzari Z, Shabkhiz F, Soudi S, Kordi MR, Hashemi SM. Combined exercise training reduces IFN-gamma and IL-17 levels in the plasma and the supernatant of peripheral blood mononuclear cells in women with multiple sclerosis. Int Immunopharmacol. 2010;10(11):1415-9. doi: 10.1016/j.intimp.2010.08.008. [PubMed: 20797460].

12. Castellano V, Patel DI, White LJ. Cytokine responses to acute and chronic exercise in multiple sclerosis. J Appl Physiol (1985). 2008;104(6):1697-702. doi: 10.1152/japplphysiol.00954.2007. [PubMed: 18388249].

13. Polman $\mathrm{CH}$, Reingold SC, Banwell B, Clanet M, Cohen JA, Filippi $M$, et al. Diagnostic criteria for multiple sclerosis: 2010 revisions to the McDonald criteria. Ann Neurol. 2011;69(2):292-302. doi: 10.1002/ana.22366. [PubMed: 21387374]. [PubMed Central: PMC3084507].

14. American College of Sports Medicine. ACSM's guidelines for exercise testing and prescription. US: Lippincott Williams \& Wilkins; 2013.

15. Heesen C, Gold SM, Hartmann S, Mladek M, Reer R, Braumann KM, et al. Endocrine and cytokine responses to standardized physical stress in multiple sclerosis. Brain Behav Immun. 2003;17(6):473-81. doi: 10.1016/s0889-1591(03)00077-1. [PubMed: 14583239].

16. Castellano V, White LJ. Serum brain-derived neurotrophic factor response to aerobic exercise in multiple sclerosis. J Neurol Sci. 2008;269(1-2):85-91. doi: 10.1016/j.jns.2007.12.030. [PubMed: 18275972].

17. Kojda G, Hambrecht R. Molecular mechanisms of vascular adaptations to exercise. Physical activity as an effective antioxidant therapy? Cardiovasc Res. 2005;67(2):187-97. doi: 10.1016/j.cardiores.2005.04.032. [PubMed: 15935334].

18. Islami D, Bischof $\mathrm{P}$, Chardonnens D. Modulation of placental vascular endothelial growth factor by leptin and hCG. Mol Hum Reprod. 2003;9(7):395-8. doi: 10.1093/molehr/gag053. [PubMed:12802046].

19. Van Craenenbroeck EM, Vrints CJ, Haine SE, Vermeulen K, Goovaerts I, Van Tendeloo VF, et al. A maximal exercise bout increases the number of circulating CD34+/KDR+ endothelial progenitor cells in healthy subjects. Relation with lipid profile. J Appl Physiol (1985). 2008;104(4):1006-13. doi: 10.1152/japplphysiol.01210.2007. [PubMed 18218912].

20. Suhr F, Brixius K, de Marees M, Bolck B, Kleinoder H, Achtzehn S, et al. Effects of short-term vibration and hypoxia during high-intensity cycling exercise on circulating levels of angiogenic regulators in humans. J Appl Physiol (1985). 2007;103(2):474-83. doi: 10.1152/japplphysiol.01160.2006. [PubMed: 17446405].

21. Shakoor E, Qassemian A, Jahromi MK, Mehrez A. Effects of 8-week aerobic exercise training on vascular endothelial growth: Factor among postmenopausal women. Int J Appl Exercise Physiol. 2016;5(4).

22. Roberts KC, Nixon C, Unthank JL, Lash JM. Femoral artery ligation stimulates capillary growth and limits training-induced increases in oxidative capacity in rats. Microcirculation. 1997;4(2):253-60. doi: 10.3109/10739689709146788. [PubMed: 9219217].

23. Deckx N, Wens I, Nuyts AH, Hens N, De Winter BY, Koppen G, et al. 12 weeks of combined endurance and resistance training reduces innate markers of inflammation in a randomized controlled clinical trial in patients with multiple sclerosis. Mediators Inflamm. 2016;2016:6789276. doi: 10.1155/2016/6789276. [PubMed: 26903712]. [PubMed Central: PMC4745915].

24. Alvarenga-Filho H, Sacramento PM, Ferreira TB, Hygino J, Abreu JEC, Carvalho SR, et al. Combined exercise training reduces fatigue and modulates the cytokine profile of T-cells from multiple sclerosis patients in response to neuromediators. J Neuroimmunol. 2016;293:91-9. doi: 10.1016/j.jneuroim.2016.02.014. [PubMed: 27049568].

25. Kjolhede T, Dalgas U, Gade AB, Bjerre M, Stenager E, Petersen T, et al. Acute and chronic cytokine responses to resistance exercise and training in people with multiple sclerosis. Scand J Med Sci Sports. 2016;26(7):824-34. doi:10.1111/sms.12504. [PubMed: 26105554].

26. Dimitrov S, Hulteng E, Hong S. Inflammation and exercise: Inhibition of monocytic intracellular TNF production by acute exercise via beta2-adrenergic activation. Brain Behav Immun. 2017;61:608. doi: 10.1016/j.bbi.2016.12.017. [PubMed: 28011264]. [PubMed Central: PMC6555138].

27. Arnett HA, Mason J, Marino M, Suzuki K, Matsushima GK, Ting JP TNF alpha promotes proliferation of oligodendrocyte progenitors and remyelination. Nat Neurosci. 2001;4(11):1116-22. doi: 10.1038/nn738. [PubMed: 11600888].

28. Peschon JJ, Torrance DS, Stocking KL, Glaccum MB, Otten C, Willis CR et al. TNF receptor-deficient mice reveal divergent roles for $\mathrm{p} 55$ and p75 in several models of inflammation.JImmunol.1998;160(2):943-52. [PubMed: 9551933].

29. Ribeiro CM, Oliveira SR, Alfieri DF, Flauzino T, Kaimen-Maciel DR, Simao ANC, et al. Tumor necrosis factor alpha (TNF-alpha) and its soluble receptors are associated with disability, disability progression and clinical forms of multiple sclerosis. Inflamm Res. 2019;68(12):1049-59. doi: 10.1007/s00011-019-01286-0. [PubMed: 31559449].

30. Gobel K, Ruck T, Meuth SG. Cytokine signaling in multiple sclerosis: Lost in translation. Mult Scler. 2018;24(4):432-9. doi: 10.1177/1352458518763094. [PubMed: 29512406].

31. Sosnoff J, Motl RW, Snook EM, Wynn D. Effect of a 4-week period of unloaded leg cycling exercise on spasticity in multiple sclerosis. NeuroRehabilitation. 2009;24(4):327-31. doi: 10.3233/NRE-2009-0486. [PubMed: 19597270]. 